\title{
Emergence of stationary multimodality under two-timescaled dichotomic noise
}

\author{
Adrián A. Budini $\odot,{ }^{1, *}$ Isaias McHardy $\odot,{ }^{2}$ Marco Nizama $\odot,{ }^{3}$ and Manuel O. Cáceres $\odot^{4}$ \\ ${ }^{1}$ Consejo Nacional de Investigaciones Científicas y Técnicas (CONICET), Centro Atómico Bariloche, \\ Avenida E. Bustillo Km 9.5, (8400) Bariloche, Argentina \\ and Universidad Tecnológica Nacional (UTN-FRBA), Fanny Newbery 111, (8400) Bariloche, Argentina \\ ${ }^{2}$ Fellowship CONICET, Centro Atómico Bariloche, 8400 Bariloche, Argentina \\ ${ }^{3}$ Departamento de Física, Universidad Nacional del Comahue and CONICET, 8300 Neuquen, Argentina \\ ${ }^{4}$ Centro Atómico Bariloche, CNEA, Instituto Balseiro, Universidad Nacional de Cuyo, and CONICET, 8400 Bariloche, Argentina
}

(Received 12 March 2020; revised manuscript received 5 May 2020; accepted 6 May 2020; published 26 May 2020)

\begin{abstract}
We study a linear Langevin dynamics driven by an additive non-Markovian symmetrical dichotomic noise. It is shown that when the statistics of the time intervals between noise transitions is characterized by two well differentiated timescales, the stationary distribution may develop multimodality (bi- and trimodality). The underlying effects that lead to a probability concentration in different points include intermittence and also a dynamical locking of realizations. Our results are supported by numerical simulations as well as by an exact treatment obtained from a Markovian embedding of the full dynamics, which leads to a third-order differential equation for the stationary distribution.
\end{abstract}

DOI: 10.1103/PhysRevE.101.052137

\section{INTRODUCTION}

The behavior of far-from-equilibrium systems has been a subject of study during recent decades. In fact, a rich variety of behaviors arises when studying the interplay between deterministic dynamical effects and external noise fluctuations [1-4]. This last ingredient may induce statistical properties that are unexpected when considering solely the deterministic dynamics.

A paradigmatic property that fluctuations may induce is multimodality, meaning that a system fluctuates with higher probability around a set of well-defined points in its phase space. Their location follows from the interplay between the deterministic (dissipative in general) system dynamics and the statistical properties of the driven fluctuations. For a wide class of systems the fluctuations can be represented by Gaussian white noises. Thus, the probability evolution can be described through a Focker-Planck dynamics [1-4]. Some examples where multimodality can be found in a stationary regime are diffusion driven by a random shear flow [5,6], and grafted polymers [7-10], just to name a few [1]. The developing of transient multimodality in Focker-Planck dynamics has also been of interest [11].

Complexity may lead to a breakdown of the Gaussian noise assumption. In fact, multimodality has also been studied beyond this approximation. For example, in Refs. [12,13] it was shown that bimodality may arise in confined Langevin dynamics driven by Lévy flights. The stationary probability concentrates in locations that do not correspond to a minimal of the potential energy. Extension of this unusual noise-induced phenomenon to asymmetric Lévy noises was performed in Ref. [14], while the emergence of multimodality under similar conditions was studied recently in Ref. [15].

\footnotetext{
*Corresponding author: adrianbudini@gmail.com
}

Dichotomic noises are a class of non-Gaussian fluctuations that are also of interest for modeling a wide class of nonequilibrium dynamics. In fact, environments switching between two well differentiated states lead to this case, which in turn may also induce bimodality [16-20]. Similarly to the previous noise statistics [12-15], the emergence of this property in stochastic Langevin dynamics driven by both dichotomic and (white) Gaussian noises has also been studied [21-23]. In particular, in Refs. [22,23] it was shown that a linear stochastic dynamics may lead to a transitory bimodality. This effect can be understood by a statistical smoothing produced by the Gaussian noise on the (transient) "front probability" induced by the dichotomic noise [24].

In the same way as previous studies based on Langevin equations (Refs. [12-15] and [21-23]), the goal of this paper is to study the emergence of stationary multimodality in linear stochastic dynamics driven by a (unique) two-timescaled dichotomic noise. Due to the linearity of the underlying dynamics this effect cannot be understood as fluctuations around fixed points of the deterministic dynamics [25-28]. In fact, we show that contrarily to previous results on linear Langevin dynamics driven by a dichotomic noise with an exponential correlation [29-33], here the interplay between dissipation and the noise timescales leads to the emergence of stationary multimodality (bi- and trimodality).

The noise transitions are defined by a renewal process [34,35]. The corresponding waiting time distribution, which gives the probability density for the elapsed time between noise transitions, is characterized here by two different timescales (biexponential behavior), in contrast to a monotonic exponential behavior. When these two timescales are widely separated, in the scale of the intrinsic dissipation time, phenomena such as intermittence and a dynamical locking of realizations emerge, which in turn are related to the emergence of multimodality in the stationary probability density. Our 
results are supported by numerical simulations. In addition, using a Markovian embedding of the total dynamics [36], an exact analytical treatment is developed. It leads to a third-order differential equation for the stationary distribution, which from a series expansion provides an exact analytical support to our main results.

The manuscript is outlined as follows. In Sec. II we introduce the stochastic Langevin dynamics and characterize the noise properties. In Sec. III, after formulating a Markovian embedding of the stochastic dynamics, we obtain a differential equation for the stationary probability density. In Sec. IV the phenomenon of noise-induced multimodality is studied. In Sec. V, we provide the Conclusions. Calculation details are provided in the Appendices.

\section{STOCHASTIC DYNAMICS}

The underlying dynamics is defined by a stochastic variable $v_{\mathrm{st}}(t)$ whose realizations follow a linear Langevin evolution,

$$
\frac{d v_{\mathrm{st}}(t)}{d t}=-\gamma v_{\mathrm{st}}(t)+\xi(t)
$$

$v_{\mathrm{st}}(t)$ can be read as the velocity of a "Brownian" particle. For simplicity, it is taken as a dimensionless variable. As usual, the constant $\gamma$ characterizes dissipation, while $\xi(t)$ is a symmetric (renewal) dichotomic noise with amplitude $\xi_{0}$. Thus, at random times it switches between the constant values $\pm \xi_{0}$. In each sojourn time interval $\left(t_{i}, t_{i+1}\right)$, where the noise amplitude is constant, the stochastic dynamics Eq. (1) can be integrated as

$$
v_{\mathrm{st}}(t)=e^{-\gamma\left(t-t_{i}\right)} v_{\mathrm{st}}\left(t_{i}\right) \pm \frac{\xi_{0}}{\gamma}\left[1-e^{-\gamma\left(t-t_{i}\right)}\right], \quad t \in\left(t_{i+1}, t_{i}\right),
$$

where the two solutions $( \pm)$ depend on the noise sign. From this expression it follows that when $\gamma\left(t_{i+1}-t_{i}\right) \gg 1$ the realizations saturate to the constant extreme values $\pm v_{0}$ with $v_{0} \equiv$ $\left(\xi_{0} / \gamma\right)$. Furthermore, it is simple to realize that when $v_{\mathrm{st}}(t)$ reaches the interval $\left(-v_{0},+v_{0}\right)$, it remains in this interval forever. Consequently, the stationary distribution associated to $v_{s t}(t)$ is restricted to that domain.

The time intervals $\Delta t_{i}=t_{i+1}-t_{i}$ are statistically independent random variables, which gives the renewal character of the evolution [34,35]. A waiting time distribution $w(t)$ $\left[t \leftrightarrow \Delta t_{i}=t_{i+1}-t_{i}\right.$ ] defines their probability density, with normalization $\int_{0}^{\infty} d t w(t)=1$. The random time intervals can explicitly be obtained from a survival probability $\Phi(t) \equiv$ $1-\int_{0}^{t} d t^{\prime} w\left(t^{\prime}\right)$. In fact, they follow from solving the equation $\Phi(t)=r$, with random numbers $r \in(0,1)$. This procedure allows us obtain the realizations $v_{s t}(t)$ after knowing $\Phi(t)$.

\section{A. Markovian dichotomic noise}

A common choice of the time interval statistics is given by an exponential function,

$$
w(t)=\phi e^{-\phi t}, \quad \Phi(t)=e^{-\phi t},
$$

where $\phi$ is a characteristic rate. As is well known [3], this statistics defines a Markovian noise dynamics, where the times at which the noise transitions happen are Poissonian distributed [34,35]. The noise correlation $C_{\xi}(t) \equiv\langle\xi(t) \xi(0)\rangle$, where $\langle\cdots\rangle$ denotes an ensemble average, reads $C_{\xi}(t)=$ $\xi_{0}^{2} \exp [-2 \phi|t|]$. Different analyses of linear Langevin dynamics driven by this kind of statistics were performed in Refs. [29-32].

\section{B. Two-timescaled non-Markovian renewal dichotomic noise}

In our approach, we consider departures with respect to an exponential waiting time distribution, which renders the noise dynamics non-Markovian. As a minimal model, we consider a biexponential structure,

$$
w(t)=p \alpha e^{-\alpha t}+q \beta e^{-\beta t}, \quad \Phi(t)=p e^{-\alpha t}+q e^{-\beta t},
$$

where the rate constants $\alpha$ and $\beta$ are arbitrary ones, while the weights $p$ and $q$ satisfy $p+q=1$.

Depending on the values or the characteristic parameters $\{p, \alpha, \beta\}$, the waiting time distribution (and survival probability) may develop strong departures with respect to an exponential behavior. As analyzed below, this feature leads to drastic changes in the statistical properties of $v_{s t}(t)$. As a matter of fact, the noise correlation $C_{\xi}(t)$ becomes a biexponential function (see Eq. (39) in Ref. [36]), which has a finite correlation time. Thus, the noise is ergodic in mean square $[1,2]$.

\section{MARKOVIAN EMBEDDING AND STATIONARY DISTRIBUTION}

Relevant statistical properties of the processes $v_{\mathrm{st}}(t)$ [Eq. (1)] can be studied through its stationary probability density $P(v)=\lim _{t \rightarrow \infty} P(v, t)$. The evolution of the timedependent probability density $P(v, t)$ can be formulated in terms of a Markovian embedding of the "velocity" dynamics, where auxiliary variables represent the noise states. For clarity we briefly review the standard Markovian embedding noise picture.

For the exponential case [Eq. (3)], we write $P(v, t)=$ $P_{+}(v, t)+P_{-}(v, t)$, where the auxiliary functions $P_{+}(v, t)$ and $P_{-}(v, t)$ satisfy (for simplicity, dependencies on $v$ and $t$ are omitted)

$$
\begin{aligned}
\frac{\partial P_{+}}{\partial t} & =\mathcal{L}_{+}\left[P_{+}\right]-\phi P_{+}+\phi P_{-}, \\
\frac{\partial P_{-}}{\partial t} & =\mathcal{L}_{-}\left[P_{-}\right]-\phi P_{-}+\phi P_{+} .
\end{aligned}
$$

The Liouville operators $\mathcal{L}_{ \pm}$are defined by their action on arbitrary functions $\Psi_{v}$ of $v$ as

$$
\mathcal{L}_{ \pm}\left[\Psi_{v}\right] \equiv-\frac{\partial}{\partial v}\left[\left(-\gamma v \pm \xi_{0}\right) \Psi_{v}\right]
$$

It is simple to realize that $P_{+}$and $P_{-}$correspond to the (joint) probability density of the stochastic variables $v_{\mathrm{st}}(t)$ and the noise $\xi(t)$. In fact, in Eq. (5), the operators $\mathcal{L}_{ \pm}$take into account the dissipative dynamics with rate $\gamma$ under external driven "forces" $\pm \xi_{0}$, while the terms proportional to the rate $\phi$ represent the noise transitions. From Eq. (5) it is possible to derive a differential equation for the stationary distribution $P(v)$, which reads (see Appendix A)

$$
\left(v^{2}-v_{0}^{2}\right) \frac{\partial P(v)}{\partial v}+2 v \frac{\gamma-\phi}{\gamma} P(v)=0 .
$$


This differential equation can be integrated, recovering the solution [see Eq. (10) below] found in previous works [29-32].

For the biexponential case [Eq. (4)] a similar treatment can be formulated. As demonstrated in Ref. [36], a "fourlevel" representation is necessary to take into account the two timescales of the noise. We write $P(v, t)=P_{\alpha,+}+P_{\beta,+}+$ $P_{\alpha,-}+P_{\beta,-}$. Thus, each noise state $( \pm)$ is split in two extra states labeled by indexes $\alpha$ and $\beta$. With adequate coupling rates between the four states, the extended (four-level) dynamic reproduces the statistics of the biexponential waiting time distribution. The auxiliary joint probability densities evolve as

$$
\begin{aligned}
& \frac{\partial P_{\alpha,+}}{\partial t}=\mathcal{L}_{+}\left[P_{\alpha,+}\right]-\alpha P_{\alpha,+}+\alpha p P_{\alpha,-}+\beta p P_{\beta,-}, \\
& \frac{\partial P_{\beta,+}}{\partial t}=\mathcal{L}_{+}\left[P_{\beta,+}\right]-\beta P_{\beta,+}+\alpha q P_{\alpha,-}+\beta q P_{\beta,-}, \\
& \frac{\partial P_{\alpha,-}}{\partial t}=\mathcal{L}_{-}\left[P_{\alpha,-}\right]-\alpha P_{\alpha,-}+\alpha p P_{\alpha,+}+\beta p P_{\beta,+}, \\
& \frac{\partial P_{\beta,-}}{\partial t}=\mathcal{L}_{-}\left[P_{\beta,-}\right]-\beta P_{\beta,-}+\alpha q P_{\alpha,+}+\beta q P_{\beta,+} .
\end{aligned}
$$

Similarly to Eq. (5), the differential operators $\mathcal{L}_{ \pm}$take into account the dissipative dynamics and the external noise "force," while the remaining terms define a master equation for the "four-level noise." Using that the probability of remaining in a given level $i=\{(\alpha, \pm),(\beta, \pm)\}$ is $\exp \left(-t \sum_{j} \phi_{j i}\right)$, where the rates are $\left\{\phi_{j i}\right\}=\{\alpha p, \alpha q, \beta p, \beta q\}$, and that the transition probability $T_{j \leftarrow i}$ between states $i$ and $j$ is $T_{j \leftarrow i}=$ $\phi_{j i} / \sum_{j^{\prime}} \phi_{j^{\prime} i}$, it is simple to realize that the biexponential statistics Eq. (4) characterizes the transitions between the two (effective) noise states defined from the probabilities $P_{+}=$ $P_{\alpha,+}+P_{\beta,+}$ and $P_{-}=P_{\alpha,-}+P_{\beta,-}$ [36].

As in the standard exponential case, from the embedding Eq. (8) it is possible to derive a differential equation for the stationary distribution $P(v)=\lim _{t \rightarrow \infty} P(v, t)=$ $\lim _{t \rightarrow \infty}\left(P_{\alpha,+}+P_{\beta,+}+P_{\alpha,-}+P_{\beta,-}\right)$. In contrast to Eq. (7), here a third-order differential equation is obtained (see Appendix A),

$f_{3}(v) \frac{\partial^{3} P(v)}{\partial v^{3}}+f_{2}(v) \frac{\partial^{2} P(v)}{\partial v^{2}}+f_{1}(v) \frac{\partial P(v)}{\partial v}+f_{0}(v) P(v)=0$.

The functions $\left\{f_{i}(v)\right\}$ are

$$
\begin{array}{ll}
f_{0}(v)=\kappa_{0} v, & f_{1}(v)=\kappa_{1} v_{0}^{2}+\kappa_{1}^{\prime} v^{2}, \\
f_{2}(v)=\kappa_{2} v\left(v^{2}-v_{0}^{2}\right), & f_{3}(v)=\kappa_{3}\left(v^{2}-v_{0}^{2}\right)^{2} .
\end{array}
$$

The auxiliary constants $\left\{\kappa_{0}, \kappa_{1}, \kappa_{1}^{\prime}, \kappa_{2}, \kappa_{3}\right\}$ are also provided in Appendix A.

A solution of Eq. (9) in terms of elementary or simple functions is unavailable. Nevertheless, in Appendix B we demonstrate that an exact series solution can consistently be found. This procedure gives us a rigorous analytical support to the next analysis.

\section{NOISE-INDUCED MULTIMODALITY}

In this section we analyze how the presence of two different timescales in the time elapsed between consecutive transitions of the noise [Eq. (4)] may lead to multimodality. As a guideline, we first analyze the exponential case [Eq. (3)].

In the Markovian noise case, the stationary probability distribution corresponding to Eq. (7) $\left[P(v) \rightarrow P_{M}(v, \phi)\right]$ is

$$
P_{M}(v, \phi)=\frac{1}{\mathcal{N}}\left[1-\left(v / v_{0}\right)^{2}\right]^{\frac{\phi}{\gamma}-1}, \quad v_{0}=\frac{\xi_{0}}{\gamma} .
$$

$\mathcal{N}$ is a normalization constant that depends on the characteristic parameters. As is well known [29-32], when $\phi / \gamma>1$, the probability is unimodal with a $\cap$-shaped form, where the probability concentrates around the origin. When $\phi / \gamma=$ 1 , the probability is constant over the domain $\left(-v_{0}, v_{0}\right)$. When $\phi / \gamma<1$, the probability assumes a $\cup$-shaped form, being concentrated in $\pm v_{0}$. The transition between the former unimodal behavior to the last bimodal behavior can be understood from the structure of the realizations [Eq. (2)]. They concentrate around the origin $(v \approx 0)$ or around the extremes $\left(v \approx \pm v_{0}\right)$ of the probability support depending on the relation between the characteristic time $1 / \phi$ of noise transitions and the dissipation characteristic time $1 / \gamma$. Below we show how extra bimodal and trimodal features arise when introducing two timescales in the waiting time distribution.

\section{A. Intermittence}

The random time interval $\Delta t=t_{i+1}-t_{i}$ [see Eq. (2)] elapsed between noise transitions can be obtained by generating a random number $r \in(0,1)$ and solving the equation $\Phi(\Delta t)=r$, where $\Phi(t)$ is the survival probability associated to the waiting time distribution $w(t)$. This numerical procedure, which is valid independently of the chosen statistics, allows us to understand how in the biexponential case [Eq. (4)] the noise $\xi(t)$ may develop intermittence, that is, a random change between two well differentiated regimes. This property, depending on dissipation, may be inherited by the variable $v_{\mathrm{st}}(t)$ leading to multimodality in $P(v)$. Both features are clearly shown in Figs. 1 and 2, respectively.

To get intermittence, we assume that the parameters defining the waiting time distribution [Eq. (4)] satisfy the inequalities

$$
\alpha \gg \beta, \quad p \simeq 1
$$

Under these conditions it is simple to realize that two timescales characterize the survival probability $\Phi(t)$. In Fig. 1 (upper panels) we plot this object jointly with the (ordinary) noise correlation $C_{\xi}(t)$ [36] for parameters satisfying the previous inequalities. In the short timescale $(\beta t \ll 1)$ it follows $\Phi(t) \simeq \Phi_{\alpha}(t) \equiv \alpha \exp [-\alpha t]$, while in the posterior regime $(\alpha t \gg 1) \Phi(t) \simeq \Phi_{\beta}(t) \equiv \beta \exp [-\beta t]$. Given that $p \simeq 1$, when generating a noise realization, most of the successive random time intervals are chosen with probability $p \simeq 1$ (approximately) with the distribution $\Phi_{\alpha}(t)$, while intervals with a statistics $\Phi_{\beta}(t)$ are rarely chosen with probability $q=1-p \simeq 0$. The changes between these two statistics lead to intermittence. This effect is clearly visible in the middle panel of Fig. 1, where a typical noise realization is shown. In fact, many successive time intervals have an average time $(1 / \alpha)$ [corresponding to $\left.\Phi_{\alpha}(t)\right]$, property that we define as an "active (A) regime," where many subsequent transitions $+\xi_{0} \leftrightarrow-\xi_{0}$ happen (almost black regions). The previous 

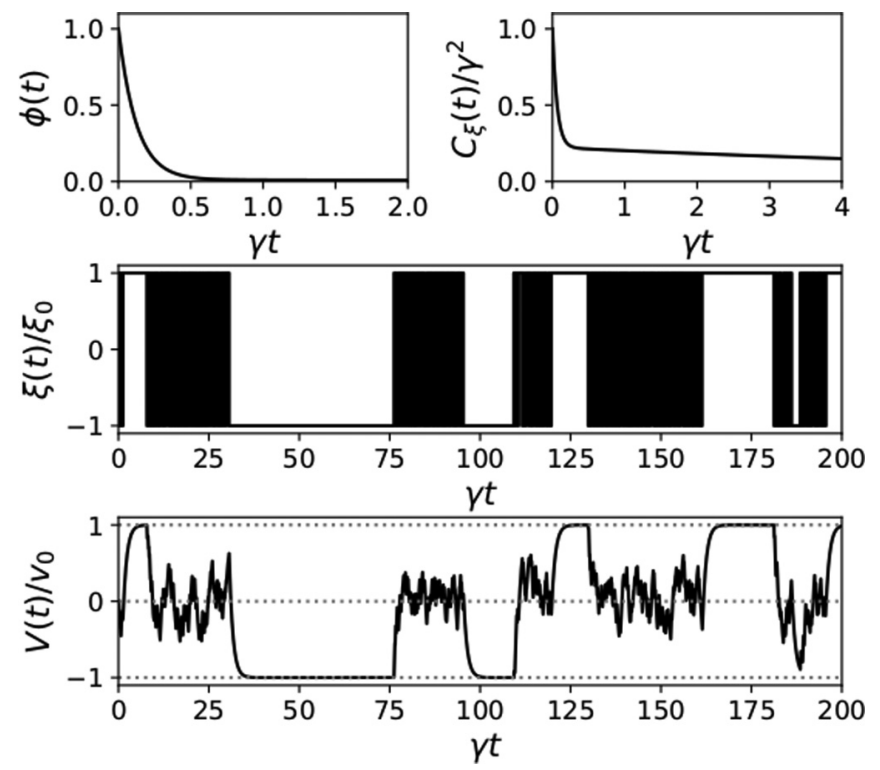

FIG. 1. Upper (left and right) panels correspond to the survival probability $\Phi(t)$ and noise correlation $C_{\xi}(t)$, respectively. Middle panel, realization of the noise $\xi(t)$. Lower panel, realization of $v_{\mathrm{st}}(t)$, where the maximal amplitude is $v_{0}=\left(\xi_{0} / \gamma\right)$. In all cases, the noise parameters are $p=0.99, \alpha / \gamma=8$, and $\beta / \gamma=0.1$, where $\gamma$ is the dissipative rate.

regime is interrupted by intervals characterized by an average time $(1 / \beta)$ [corresponding to $\Phi_{\beta}(t)$ ], being associated to an "inactive (I) regime," where more than one transition rarely happens. Given that $p \simeq 1$, after an inactive regime is more likely that a new active regime begins, leading to noise intermittence.

The durations of the active and inactive noise regimes are random variables (see Fig. 1). Their probability densities, denoted as $W_{A}(T)$ and $W_{I}(T)$, respectively, cannot be obtained in an exact way. Nevertheless, when $p \simeq 1$ and $\alpha \gg \beta$ [Eq. (11)] they can be approached in the following way. Denoting the Laplace transform $(u \leftrightarrow t)$ with a hat symbol $\left[\hat{f}(u) \equiv \int_{0}^{\infty} d t e^{-u t} f(t)\right]$, we write

$$
\begin{aligned}
\hat{W}_{A}(u) p \simeq \sum_{n=1}^{\infty}\left[p \hat{w}_{\alpha}(u)\right]^{n}(1-p), \\
\hat{W}_{I}(u) q \simeq \sum_{n=1}^{\infty}\left[q \hat{w}_{\beta}(u)\right]^{n}(1-q),
\end{aligned}
$$

where $w_{\alpha}(t) \equiv \alpha \exp [-\alpha t]$ and $w_{\beta}(t) \equiv \beta \exp [-\beta t]$. The symbol $\simeq$ denotes that these expressions are valid under the conditions Eq. (11). We considered that a random time interval in the active (inactive) regime can be obtained as the addition of an arbitrary number of consecutive events, each one occurring with probability $p[(1-p)]$ and having a duration given by the waiting time distribution $w_{\alpha}(t)\left[w_{\beta}(t)\right]$. This approximation, which is valid under the conditions Eq. (11), explains the convolution structure (in the time domain) of Eq. (12), that is, the contributions $p^{n} \hat{w}_{\alpha}^{n}(u)\left[q \hat{w}_{\beta}(u)\right]^{n}$. The extra factor $(1-p)[(1-q)]$ takes into account the end of an active (inactive) period. Similarly, the factors accompanying $\hat{W}_{A}(u)$ and $\hat{W}_{B}(u)$ take into account that the active and inactive
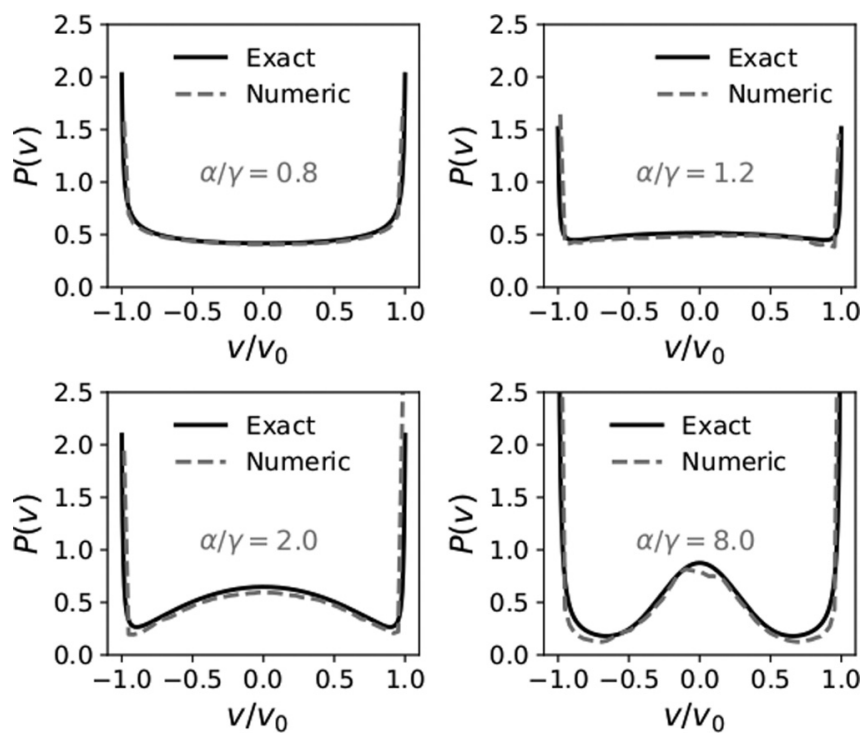

FIG. 2. Stationary probability density $P(v)$ in the intermittence regime. Both, the series solution (full line) and the numerical one (dashed line) are shown. In all cases the parameters are $p=$ $0.99, \beta / \gamma=0.1$, while $\alpha / \gamma=0.8,1.2,2$, and 8 .

periods begin with probability $p$ and $q$ after the end of the complementary period, respectively.

We notice that $\hat{W}_{A}(u)$ and $\hat{W}_{B}(u)$ can also be read as moment generating functions [37]. Nevertheless, their (sum) structures in Eq. (12) were not derived from this property. They represent a sum over all possible underlying realizations. On the other hand, using that $\hat{w}_{\alpha}(u)=$ $\alpha /(\alpha+u)$ and $\hat{w}_{\beta}(u)=\beta /(\beta+u)$, from Eq. (12) straightforwardly it follows $W_{A}(T) \simeq T_{A}^{-1} \exp \left[-T / T_{A}\right]$ and $W_{I}(T) \simeq$ $T_{I}^{-1} \exp \left[-T / T_{I}\right]$. The parameters $T_{A}$ and $T_{I}$ are

$$
T_{A}=\frac{1}{(1-p) \alpha}, \quad T_{I}=\frac{1}{p \beta},
$$

which in turn define the corresponding average times $T_{A / I}=$ $\int_{0}^{\infty} d T W_{A / I}(T) T$. These estimations are valid under the conditions defined by Eq. (11).

The previous analysis explains how, under the conditions (11), the noise may develop intermittence. Now we search for extra conditions that guarantees that this phenomenon is inherited by the process $v_{s t}(t)$ [Eq. (1)]. We found that a sufficient condition is given by the inequalities

$$
\frac{1}{\gamma} \ll T_{A}, \quad \frac{1}{\gamma} \ll T_{I} .
$$

Realizations of $v_{s t}(t)$ fulfilling these conditions are shown in Fig. 1 (lower panel) which clearly develops the phenomenon of intermittence.

The conditions Eq. (14) have the following meaning. They imply that in the time intervals of both the active and inactive noise regimes the statistics of the variable $v_{s t}(t)$, due to dissipation, reaches a stationary state. The corresponding stationary probability densities depend on which state (active versus inactive) the noise is. In the active and inactive regimes the stationary probability density can, respectively, be approximated by $P_{M}(v, \alpha)$ and $P_{M}(v, \beta)$, that is, Eq. (10) 

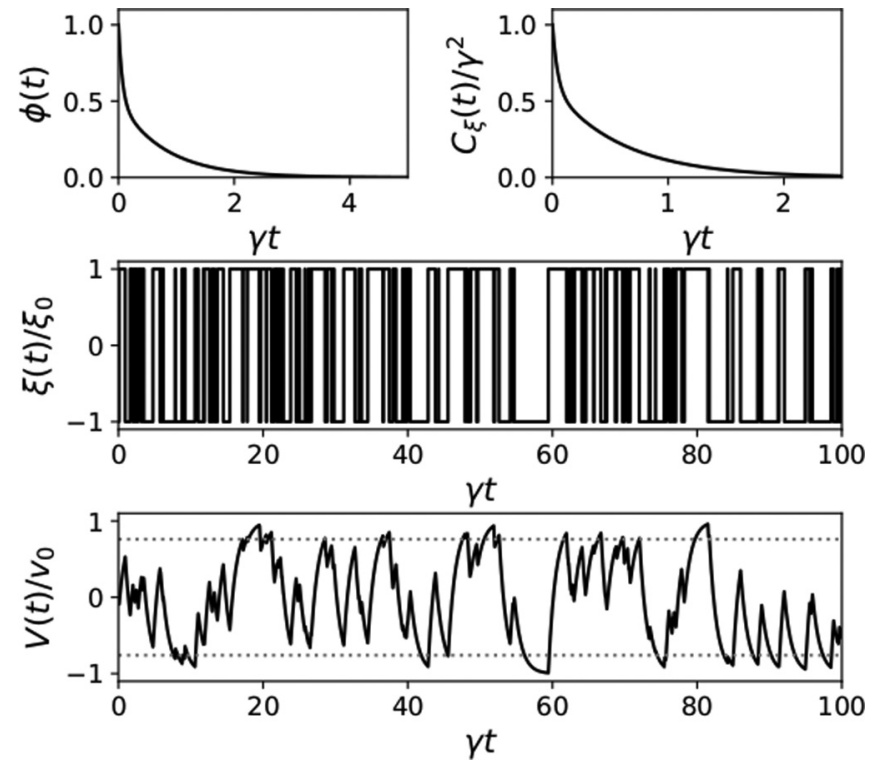

FIG. 3. Upper (left and right) panels correspond to the survival probability $\Phi(t)$ and noise correlation $C_{\xi}(t)$, respectively. Middle panel, realization of the noise $\xi(t)$. Lower panel, stochastic realization of $v_{\mathrm{st}}(t)$. The horizontal dashed lines correspond to $v_{\max }$ [Eq. (21)]. In all cases, the noise parameters are $p=0.5, \alpha / \gamma=15$, and $\beta / \gamma=1.25$.

with rates $\alpha$ and $\beta$. Given the ergodicity [38] of the noise, the full stationary probability distribution $P(v)$ can then be approximated as

$$
P(v) \simeq \frac{T_{A}}{T_{A}+T_{I}} P_{M}(v, \alpha)+\frac{T_{I}}{T_{A}+T_{I}} P_{M}(v, \beta) .
$$

Consistently with ergodicity, the weights $T_{A} /\left(T_{A}+T_{I}\right)$ and $T_{I} /\left(T_{A}+T_{I}\right)$ correspond to the fraction of time that the noise is found in the active and inactive regimes, respectively. Given that $P_{M}(v, \alpha)$ and $P_{M}(v, \beta)$ depart considerably from each other, and given the significance of the weights, Eq. (15) demonstrates that the conditions defined by Eq. (14) lead in fact to intermittence in the realizations $v_{\mathrm{st}}(t)$, that is, a random change between two different dynamical regimes.

Depending on the characteristic parameters, the (approximated) stationary state (15) may concentrate in different ways, leading to multimodality. This property is confirmed in Fig. 2. We show different stationary states $P(v)$, each one corresponding to different characteristic parameters fulfilling conditions Eqs. (11) and (14). A change between two points of accumulations to three ones, that is, a transition between bimodality to trimodality, is clearly observed. The plots correspond to the exact series solutions of Eq. (9) jointly with numerical ones [39]. In the scale of the plots both kinds of results are almost indistinguishable. This feature is also valid for the approximate solutions given by Eq. (15).

The transition between bimodality to trimodality shown in Fig. 2 can be understood by analyzing the parameters constraint defined by Eq. (14) and the expression Eq. (13). From the definition of $T_{I}$ it follows $\beta / \gamma \ll(1 / p) \simeq 1$. Thus, taking into account the solution Eq. (10), the contribution $P_{M}(v, \beta)$ in Eq. (15) always accumulates in the extreme values $v= \pm v_{0}$. However, from $T_{A}$ it follows the condition
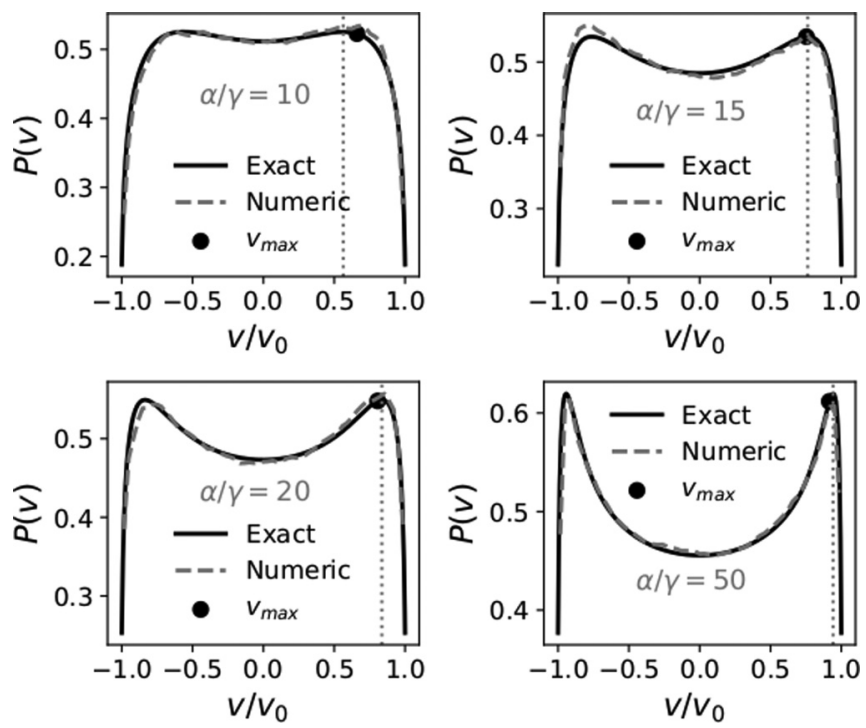

FIG. 4. Stationary probability density $P(v)$ in the dynamical locking regime. Both the series solution (full line) and numerical one (dashed line) are shown. In all cases the parameters are $p=$ $0.5, \beta / \gamma=1.25$, while $\alpha / \gamma=10,15,20$, and 50. The vertical dashed lines indicate the positions of the probability extremum.

$\alpha / \gamma \ll 1 /(1-p)$. To fulfill this inequality it may happen that $\alpha / \gamma<1$, but alternatively it may also be the case that $\alpha / \gamma>1$. These two different conditions explain the transition between bimodality to trimodality. In fact, in the first case the contribution $P_{M}(v, \alpha)$ concentrates in the extreme values $v= \pm v_{0}$ (leading to bimodality), while in the second case it concentrates around the origin $v=0$ (leading to trimodality). The realization of $v_{\mathrm{st}}(t)$ shown in Fig. 1 corresponds to this last case.

\section{B. Dynamical locking of realizations}

The bimodality that arises in the Markovian noise case [Eq. (10)] follows from a probability accumulation that only happens in the extreme values $v= \pm v_{0}$. Here, we find that the biexponential correlated noise may lead to bimodality that occurs inside the domain $\left(-v_{0},+v_{0}\right)$.

As in the case of intermittence, we consider that the survival probability is characterized by two well differentiated timescales. We assume

$$
\alpha \gg \beta \gtrsim \gamma, \quad p \simeq 1 / 2
$$

In Fig. 3 (upper panels) we show the survival probability and noise correlation under these conditions. In the middle and lower panels we also show a realization of the noise $\xi(t)$ and $v_{s t}(t)$, respectively. In contrast to intermittence, where $p \simeq 1$, here both timescales have almost the same weights, $p \simeq 1 / 2$. Thus, each timescale is selected with almost equal probability, which implies that intermittence does not develop. Hence, approximation Eq. (15) breaks down. In fact, in Fig. 4 we show a set of stationary distributions, which depending on the parameter values develop an increasing bimodal behavior in the interior of the domain $\left(-v_{0},+v_{0}\right)$. This feature, as well as that shown in Fig. 2, is completely oblivious to the Markovian noise case. 
Consistently with the bimodality shown in Fig. 4, the realizations of $v_{\mathrm{st}}(t)$ [Fig. 3] (statistically) concentrate around two values $\pm\left|v_{\max }\right|$, which in turn coincides with the position of the probability peaks. Below, we describe the mechanism that determines the value $\left|v_{\max }\right|$.

We study the stochastic variation of $v_{\mathrm{st}}(t)$ between two consecutive noise transitions, that is $\Delta v_{\mathrm{st}}^{ \pm} \equiv v_{\mathrm{st}}\left(t_{i+1}\right)-v_{\mathrm{st}}\left(t_{i}\right)$, where $( \pm)$ corresponds to the noise sign at the beginning of the time interval. From Eq. (2) we obtain

$$
\Delta v_{\mathrm{st}}^{ \pm}=\left[-v_{\mathrm{st}}\left(t_{i}\right) \pm v_{0}\right]\left(1-e^{-\gamma \Delta t}\right), \quad \Delta t=t_{i+1}-t_{i}
$$

We notice that $\Delta v_{\mathrm{st}}^{ \pm}$depends on the starting point $v_{\mathrm{st}}\left(t_{i}\right)$ and $\Delta t$. The interplay between the statistical properties of these two random variables leads to bimodality. The underlying mechanism is named as a dynamical locking of realizations. It can be understood by characterizing the ensemble (averaged) properties of $\Delta v_{\mathrm{st}}^{ \pm}$.

From Eq. (4) and the conditions Eq. (16), the successive values of $\Delta t$ can be approximated as follows. The time interval $\Delta t$ is successively chosen with probability $p \simeq 1 / 2$ with the probability density $w_{\alpha}(t)=\alpha \exp [-\alpha t]$, or complementarily with probability $q \simeq 1 / 2$ with the density $w_{\beta}(t)=$ $\beta \exp [-\beta t]$. Thus, we conjecture that the probability concentration of $P(v)$ happens at an initial condition $v_{\mathrm{st}}\left(t_{i}\right) \rightarrow v_{\max }$ such that the following statistical condition is satisfied:

$$
q\left\langle\Delta v_{\mathrm{st}}^{ \pm}\right\rangle_{\beta}=p\left\langle\Delta v_{\mathrm{st}}^{\mp}\right\rangle_{\alpha}
$$

The subindexes $\alpha$ and $\beta$ indicate that contributions proportional to $\Delta t$ are averaged with $w_{\alpha}(t)$ and $w_{\beta}(t)$, respectively, which in turn justifies the weights $p$ and $q$. Explicitly, from Eq. (17) we get

$\left|-v_{\max } \pm v_{0}\right| q\left\langle 1-e^{-\gamma \Delta t}\right\rangle_{\beta}=\left|-v_{\max } \mp v_{0}\right| p\left\langle 1-e^{-\gamma \Delta t}\right\rangle_{\alpha}$.

In this way, $v_{\max }$ defines the position where the (average) displacement induced by one timescale (denoted with the subindexes $\alpha$ and $\beta$ ) equilibrates the (average) displacement corresponding to the other timescale. Notice that both kinds of displacements are in opposite directions $( \pm \leftrightarrow \mp)$, which justifies the name of the effect. Using that each timescale is exponentially distributed, $\left\langle 1-e^{-\gamma \Delta t}\right\rangle_{\phi}=\int_{0}^{\infty} d t\left(\phi e^{-\phi t}\right)$ $\left(1-e^{-\gamma t}\right)$, it follows

$$
\left\langle 1-e^{-\gamma \Delta t}\right\rangle_{\beta}=\frac{\gamma}{\gamma+\beta}, \quad\left\langle 1-e^{-\gamma \Delta t}\right\rangle_{\alpha}=\frac{\gamma}{\gamma+\alpha} .
$$

Thus, from Eq. (19) we obtain

$$
v_{\max }= \pm v_{0}\left|\frac{\frac{q}{\gamma+\beta}-\frac{p}{\gamma+\alpha}}{\gamma+\beta}+\frac{p}{\gamma+\alpha}\right|
$$

This result provides an estimation for the location of points of maximal probability in the stationary state $P(v)$.

During a single realization, given that $p \simeq 1 / 2$, consecutive time intervals $\Delta t$ may be chosen with the same statistics [ $w_{\alpha}(t)$ or $\left.w_{\beta}(t)\right]$, a situation not considered in the previous deduction. In addition, $v_{\mathrm{st}}\left(t_{i}\right)$ may depart considerably from $v_{\text {max }}$, which invalidates the replacement $v_{\mathrm{st}}\left(t_{i}\right) \rightarrow v_{\text {max }}$. Nevertheless, we consider that these extra statistical components lead to a small modification to the previous estimation. In fact, as shown in Fig. 4, Eq. (21) provides an excellent approximation for the peak positions, which shows the consistence of the previous analysis and statistical explanation.

Contrary to the case of intermittence [Eq. (15)], here it is not easy to derive a simple analytical approximation for the full stationary distribution $P(v)$. Nevertheless, we propose the following ansatz, which is also written in terms of the stationary distribution corresponding to the Markovian noise case [Eq. (10)]. Given that $\alpha \gg \gamma$ [Eq. (16)] the stationary distribution $P_{M}(v, \alpha)$ is almost a (bounded) Gaussian distribution, while from the condition $\beta \gtrsim \gamma$ it follows that $P_{M}(v, \beta)$ is almost constant in all the domain while falling abruptly to a null value at the boundaries $\pm v_{0}$. We propose to approach the bimodal structure of $P(v)$ in terms of these distributions as follows:

$$
P(v) \simeq w_{f} P_{M}(v, \beta)+\frac{\left(1-w_{f}\right)}{2} \sum_{s= \pm 1} P_{M}\left(\frac{v-s v_{f}}{1+\left(v_{f} / v_{0}\right)}, \alpha\right)
$$

Here, $w_{f} \in(0,1)$ and $0<v_{f}<v_{0}$ are fitting parameters chosen such that the maximal values of $P(v)$ are located at $\pm\left|v_{\text {max }}\right|$ [Eq. (21)]. We found that this fitting is almost indistinguishable from the series solution and numerical simulations for almost all range of parameters values where the present bimodal states emerge.

\section{Extra dynamical regimes}

Besides the previous two cases, there exist other parameter regimes that lead to stationary distributions that cannot be obtained with an exponential correlated noise. For example, when $\beta=\gamma, 1<\alpha / \gamma \lesssim 2$, and $p=1 / 2$, the stationary probability density can be fit as $P(v) \simeq w_{f} P_{M}(v, \alpha)+(1-$ $\left.w_{f}\right) P_{M}(v, \beta)$, where the weight $w_{f} \in(0,1)$ is a fitting parameter. Given that $\beta=\gamma$, it follows that $P_{M}(v, \beta)=1 /\left(2 v_{0}\right)$, while the condition $1<\alpha / \gamma \lesssim 2$ implies that $P_{M}(v, \alpha)$ assumes a $\cap$ form. Thus, this unimodal form of $P(v)$ satisfies $P\left( \pm v_{0}\right) \neq 0$, a property that is beyond the functional forms of the solution Eq. (10). In addition, when $\beta=\gamma, \alpha / \gamma \gtrsim 10$, and $p=1 / 2, P(v)$ assumes a $\cup$ form, which in contrast to the solution Eq. (10) does not diverge at $v= \pm v_{0}, P\left( \pm v_{0}\right)<\infty$. From the third-order differential equation [Eq. (9)], it is simple to realize that a constant distribution, $P(v)=1 /\left(2 v_{0}\right)$, is also a solution for especial parameter values $\{p, \alpha, \beta\}$. Nevertheless, in contrast to the case of Eq. (10), the underlying noise fluctuations are non-Markovian. In general, the Markovian noise case is trivially recovered by taking in Eq. (4) $\alpha=\beta$ and also taking $p=1$ or $p=0$.

\section{SUMMARY AND CONCLUSIONS}

We studied the stationary probability density of a linear Langevin dynamics driven by a symmetrical renewal dichotomic noise characterized by two timescales. The statistics of the time interval between noise transitions is defined by a biexponential waiting time distribution. Depending on its characteristic parameters this function may develop two well-differentiated timescales. We found that the interplay between the noise timescales and the intrinsic dissipation 
timescale may lead to multimodality (bi- and trimodality), that is, the stationary distribution concentrates in different points. These features of the stationary probability density cannot be induced by noise fluctuations characterized by a single timescale.

When one of the two noise timescales has an almost vanishing weight, the noise realizations become intermittent, switching between active and inactive regimes. If in addition the system dissipation time defines the minor timescale of the problem, then the stationary probability may concentrate around the origin and also at the extremes values of the natural domain, leading to a transition between bimodality to trimodality. Furthermore, we found that the non-Markovian character of the driven noise may lead to bimodality whose maximal values, in contrast to the Markovian noise case, develop inside the domain of the stationary probability density. This feature arises when both noise timescales are smaller than the dissipation time, having both of them almost the same weight. The underlying effect can be understood as a dynamical locking of realizations. This effect consists in the equilibration, for each noise timescale, of the average (statedependent) system displacements.

Our conclusions were supported by numerical simulations as well as from an exact description based on a third-order differential equation fulfilled by the stationary distribution. This result was obtained from a Markovian embedding of the full stochastic dynamics.

The present results demonstrate that linear stochastic dynamics may develop characteristics (such as multimodality) typical of nonlinear dynamics. The main ingredients are dissipation and dichotomic noise transitions characterized by a minimal set of two timescales. The interplay between these components may provide an alternative frame for understanding multimodality in far-from-equilibrium (dissipative) systems coupled to multi-state environments characterized by different internal switching rates.

\section{ACKNOWLEDGMENTS}

A.A.B. thanks Mariano Bonifacio for a critical reading of the manuscript as well as support from CONICET, Argentina. M.O.C. gratefully acknowledge support received from CONICET, Grant No. PIP 112-261501-00216 CO, Argentina.

\section{APPENDIX A: DIFFERENTIAL EQUATION FOR THE STATIONARY PROBABILITY DENSITY}

In this Appendix we derive the differential equation that the stationary distribution $P(v)$ satisfies. As in previous contributions [29-32], given the intrinsic dissipative nature of the stochastic dynamics (with timescale $1 / \gamma$ ) and the finite correlation time of the noise, we assume that the obtained partial differential equations [Eqs. (5) and (8)] in a long-time regime $(t \rightarrow \infty)$ define the stationary state solution.

In the exponential correlated noise case [Eq. (3)], from the auxiliary joint probability distributions $P_{+}$and $P_{-}$[Eq. (5)] we define the functions

$$
P(v) \equiv \lim _{t \rightarrow \infty}\left(P_{+}+P_{-}\right), \quad Q(v) \equiv \lim _{t \rightarrow \infty}\left(P_{+}-P_{-}\right) .
$$

Taking the limit $t \rightarrow \infty$ in Eq. (5), we obtain

$$
\begin{aligned}
& 0=\frac{\partial}{\partial v}\left[\gamma v P(v)-\xi_{0} Q(v)\right], \\
& 0=\frac{\partial}{\partial v}\left[\gamma v Q(v)-\xi_{0} P(v)\right]-2 \phi Q(v) .
\end{aligned}
$$

The first equation can be solved as $\gamma v P(v)-\xi_{0} Q(v)=c$. After integration in the domain $v \in\left(-v_{0}, v_{0}\right)$ it follows $c=0$, which implies $Q(v)=\left(v / v_{0}\right) P(v), \quad v_{0}=\xi_{0} / \gamma$. Reinserting this solution in the second equation, for $P(v)$ we get the differential equation

$$
\left(v^{2}-v_{0}^{2}\right) \frac{\partial P(v)}{\partial v}+2 v \frac{\gamma-\phi}{\gamma} P(v)=0,
$$

which recovers Eq. (7).

In the biexponential case [Eq. (4)] more auxiliary functions are necessary. From Eq. (8), and similarly to the previous case, we define the functions

$$
\begin{aligned}
P(v) & \equiv \lim _{t \rightarrow \infty}\left(P_{\alpha,+}+P_{\beta,+}+P_{\alpha,-}+P_{\beta,-}\right), \\
Q(v) & \equiv \lim _{t \rightarrow \infty}\left(P_{\alpha,+}+P_{\beta,+}-P_{\alpha,-}-P_{\beta,-}\right), \\
M(v) & \equiv \lim _{t \rightarrow \infty}\left(P_{\alpha,+}-P_{\beta,+}-P_{\alpha,-}+P_{\beta,-}\right), \\
N(v) & \equiv \lim _{t \rightarrow \infty}\left(P_{\alpha,+}-P_{\beta,+}+P_{\alpha,-}-P_{\beta,-}\right) .
\end{aligned}
$$

From Eq. (8), we get a set of linear differential equations for the new variables,

$$
\begin{gathered}
0=\frac{\partial}{\partial v}\left[\gamma v P(v)-\xi_{0} Q(v)\right], \\
0=\frac{\partial}{\partial v}\left[\gamma v Q(v)-\xi_{0} P(v)\right]-\mu_{+} Q(v)-\mu_{-} M(v), \\
0=\frac{\partial}{\partial v}\left[\gamma v M(v)-\xi_{0} N(v)\right]-\lambda_{+} M(v)-\lambda_{-} Q(v), \\
0=\frac{\partial}{\partial v}\left[\gamma v N(v)-\xi_{0} M(v)\right]-\eta_{+} N(v)-\eta_{-} P(v),
\end{gathered}
$$

where for shortening the expressions we defined the parameters $\mu_{ \pm} \equiv(\alpha \pm \beta), \lambda_{ \pm} \equiv(\alpha p \pm \beta q)$, and $\eta_{ \pm} \equiv(\alpha q \pm \beta p)$. This system of equations can be solved as follows: (i) obtain $Q(v)$ as a function of $P(v)$ from Eq. (A5), and replace the solution into (A6); (ii) get $M(v)$ as a function of $P(v)$ and replace into (A7); (iii) get the function $(\partial / \partial v) N(v)$ as a function of $P(v)$ from (A7); (iv) finally, take the derivative of Eq. (A8), which leads to a third-order differential equation with polynomial coefficient functions [Eq. (9)],

$$
f_{3}(v) \frac{\partial^{3} P(v)}{\partial v^{3}}+f_{2}(v) \frac{\partial^{2} P(v)}{\partial v^{2}}+f_{1}(v) \frac{\partial P(v)}{\partial v}+f_{0}(v) P(v)=0
$$

The auxiliary functions are

$$
\begin{array}{ll}
f_{0}(v)=\kappa_{0} v, & f_{1}(v)=\kappa_{1} v_{0}^{2}+\kappa_{1}^{\prime} v^{2}, \\
f_{2}(v)=\kappa_{2} v\left(v^{2}-v_{0}^{2}\right), & f_{3}(v)=\kappa_{3}\left(v^{2}-v_{0}^{2}\right)^{2},
\end{array}
$$


with constants

$$
\begin{aligned}
\kappa_{0}= & \left(3 \gamma-\eta_{+}\right)\left[4 \gamma^{2}-\lambda_{-} \mu_{-}+\lambda_{+} \mu_{+}-2 \gamma\left(\lambda_{+}+\mu_{+}\right)\right] / \gamma, \\
\kappa_{1}= & -8 \gamma^{2}-\eta_{+} \lambda_{+}-\eta_{-} \mu_{-}+\gamma\left[\eta_{+}+2\left(\lambda_{+}+\mu_{+}\right)\right], \\
\kappa_{1}^{\prime}= & 24 \gamma^{2}+\eta_{+} \lambda_{+}-\lambda_{-} \mu_{-}+\left(\eta_{+}+\lambda_{+}\right) \mu_{+} \\
& -\gamma\left[5 \eta_{+}+6\left(\lambda_{+}+\mu_{+}\right)\right], \\
\kappa_{2}= & \gamma\left(10 \gamma-\eta_{+}-\lambda_{+}-\mu_{+}\right), \quad \kappa_{3}=\gamma^{2} .
\end{aligned}
$$

The previous third-order differential equation is consistent with the symmetry $P(+v)=P(-v)$.

\section{APPENDIX B: SERIES SOLUTION FOR THE STATIONARY PROBABILITY}

To solve Eq. (A9), $P(v)$ is written as a power series,

$$
P(v)=\sum_{m=0}^{\infty} c_{m} v^{2 m}
$$

Given the symmetry $P(+v)=P(-v)$, only even terms are considered. After replacing Eq. (B1) into Eq. (A9), we obtain a recurrence relation for the unknown coefficients $c_{m}$,

$$
g_{m}^{(0)} c_{m}=g_{m}^{(1)} c_{m-1}+g_{m}^{(2)} c_{m-2},
$$

where the auxiliary functions are

$$
\begin{aligned}
& g_{m}^{(0)}=v_{0}^{4} \kappa_{3}[4 m(m-1)(2 m-1)], \\
& g_{m}^{(1)}=v_{0}^{2}(2 m-2)\left\{\kappa_{1}+(2 m-3)\left[\kappa_{2}-4(m-2) \kappa_{3}\right]\right\}, \\
& g_{m}^{(2)}=\kappa_{0}+2(m-2)\left\{(5-2 m)\left[\kappa_{2}-2(m-3) \kappa_{3}\right]+\kappa_{1}^{\prime}\right\} .
\end{aligned}
$$

The recurrence relation Eq. (B2) is valid for $m \geqslant 2$. Thus, consistently with the order of the differential equation, the first two coefficients $c_{0}$ and $c_{1}$ are undetermined. Given that $c_{0}=\left.P(v)\right|_{v=0}$ and $c_{1}=\left.(1 / 2)\left(d^{2} / d v^{2}\right) P(v)\right|_{=0}$, their mutual dependence should be obtained from Eqs. (A5) to (A8) and their derivatives evaluated in $v=0$. Nevertheless, using that $P(v)=P(-v), \quad Q(v)=-Q(-v), \quad M(v)=-M(-v)$, and $N(v)=N(-v)$, it follows that Eqs. (A6) and (A7) are satisfied as identities in $v=0$. A similar degeneracy arises in the derivatives. Thus, we are challenged to search for an alternative closure condition.

First, we notice that the previous auxiliary functions satisfy the normalizations

$$
\int_{-v_{0}}^{+v_{0}} P(v) d v=1, \quad \int_{-v_{0}}^{+v_{0}} N(v) d v=\frac{\beta p-\alpha q}{\alpha q+\beta p},
$$

while $\int_{-v_{0}}^{+v_{0}} Q(v) d v=\int_{-v_{0}}^{+v_{0}} M(v) d v=0$. These integral relations follow from the definitions Eq. (A4) and performing the integral of the dynamics Eq. (8) in the long-time limit, which deliver the stationary probability of each state of the embedding.

Second, the function $N(v)$, which is an even function, can also be written as a series expansion,

$$
N(v)=\sum_{m=0}^{\infty} w_{m} v^{2 m}
$$

The coefficients $\left\{w_{n}\right\}$ can be obtained as a function of the coefficients $\left\{c_{n}\right\}$ of $P(v)$ [Eq. (B1)]. By solving $M(v)$ from Eq. (A6), and inserting the solution into Eq. (A8), we get the relation

$$
h_{m}^{(0)} w_{m}=h_{m}^{(2)} c_{m+1}+h_{m}^{(1)} c_{m}
$$

where the auxiliary functions are

$$
\begin{aligned}
& h_{m}^{(2)}=-2 \xi_{0}^{2}(2 m+1)(m+1), \\
& h_{m}^{(1)}=2 \gamma^{2}(2 m+1)(m+1)-\gamma \mu_{+}(2 m+1)+\mu_{-} \eta_{-}, \\
& h_{m}^{(0)}=\left[\gamma(2 m+1)-\eta_{+}\right] \mu_{-} .
\end{aligned}
$$

Finally, imposing the normalization defined by Eq. (B3) into the series expression Eqs. (B1) and (B4), it follows

$$
\sum_{m=0}^{\infty} \frac{v_{0}^{2 m+1} c_{m}}{2 m+1}=\frac{1}{2}, \quad \sum_{m=0}^{\infty} \frac{v_{0}^{2 m+1} w_{m}}{2 m+1}=\frac{1}{2} \frac{\beta p-\alpha q}{\alpha q+\beta p} .
$$

Given that all coefficients $\left\{c_{m}\right\}$ and $\left\{w_{m}\right\}$ can be written in terms of $c_{0}$ and $c_{1}$, the previous equalities define a set of two equations that allow to find the two indeterminate coefficients.
[1] W. Horsthemke and R. Lefever, Noise-induced Transitions (Springer-Verlag, Berlin 1984).

[2] C. W. Gardiner, Handbook of Stochastic Methods (SpringerVerlag, Berlin, 1985)

[3] N. G. van Kampen, Stochastic Process in Physics and Chemistry, 2nd ed. (North-Holland, Amsterdam, 1992).

[4] M. O. Cáceres, Nonequilibrium Statistical Physics with Application to Disordered Systems (Springer, Berlin, 2017).

[5] E. Ben-Naim, S. Redner, and D. Ben-Avraham, Bimodal diffusion in power-law shear flows, Phys. Rev. A 45, 7207 (1992).

[6] D. ben-Avraham, F. Leyvraz, and S. Redner, Superballistic motion in a "random-walk" shear flow, Phys. Rev. A 45, 2315 (1992).

[7] A. Dhar and D. Chaudhuri, Triple Minima in the Free Energy of Semiflexible Polymers, Phys. Rev. Lett. 89, 065502 (2002).

[8] G. Lattanzi, T. Munk, and E. Frey, Transverse fluctuations of grafted polymers, Phys. Rev. E 69, 021801 (2004).
[9] P. Benetatos, T. Munk, and E. Frey, Bimodality in the transverse fluctuations of a grafted semiflexible polymer and the diffusionconvection analog: An effective-medium approach, Phys. Rev. E 72, 030801(R) (2005).

[10] F. F. Semeriyanov and S. Stepanow, Bimodal distribution function of a three-dimensional wormlike chain with a fixed orientation of one end, Phys. Rev. E 75, 061801 (2007).

[11] J. Iwaniszewski, Transient multimodality in the presence of potential fluctuations, Phys. Rev. E 78, 021140 (2008).

[12] A. V. Chechkin, J. Klafter, V. Yu. Gonchar, R. Metzler, and L. V. Tanatarov, Bifurcation, bimodality, and finite variance in confined Lévy flights, Phys. Rev. E 67, 010102(R) (2003).

[13] A. V. Chechkin, V. Y. Gonchar, J. Klafter, R. Metzler, and L. V. Tanatarov, Lévy flights in a steep potential well, J. Stat. Phys. 115, 1505 (2004). 
[14] B. Dybiec, E. Gudowska-Nowak, and I. M. Sokolov, Stationary states in Langevin dynamics under asymmetric Lévy noises, Phys. Rev. E 76, 041122 (2007).

[15] M. Ciésla, K. Capała, and B. Dybiec, Multimodal stationary states under Cauchy noise, Phys. Rev. E 99, 052118 (2019).

[16] P. G. Hufton, Y. T. Lin, T. Galla, and A. J. McKane, Intrinsic noise in systems with switching environments, Phys. Rev. E 93, 052119 (2016).

[17] G. C. P. Innocentini, S. Guiziou, J. Bonnet, and O. Radulescu, Analytic framework for a stochastic binary biological switch, Phys. Rev. E 94, 062413 (2016).

[18] B. Houchmandzadeh and M. Vallade, Exact results for a noiseinduced bistable system, Phys. Rev. E 91, 022115 (2015).

[19] T. Biancalani, L. Dyson, and A. J. McKane, Noise-Induced Bistable States and Their Mean Switching Time in Foraging Colonies, Phys. Rev. Lett. 112, 038101 (2014).

[20] A. A. Budini, Large deviations of ergodic counting processes: A statistical mechanics approach, Phys. Rev. E 84, 011141 (2011); Fluctuation relations with intermittent non-Gaussian variables, 84, 061118 (2011).

[21] B. Dybieca and L. Schimansky-Geierb, Emergence of bimodality in noisy systems with single-well potential, Eur. Phys. J. B 57, 313 (2007).

[22] H. Calisto, M. Bologna, and K. J. Chandía, Forced dichotomic diffusion in a viscous media, Eur. Phys. J. B 90, 24 (2017).

[23] K. Malakar, V. Jemseena, A. Kundu, K. V. Kumar, S. Sabhapandit, S. N. Majumdar, S. Redner, and A. Dhar, Steady state, relaxation, and first-passage properties of a run-andtumble particle in one-dimension, J. Stat. Mech. (2018) 043215.

[24] A. A. Budini and M. O. Cáceres, The generalized Wiener process II: Finite systems, J. Phys. A: Math. Gen. 32, 4005 (1999).

[25] A. Ichiki, Y. Tadokoro, and M. I. Dykman, Singular response of bistable systems driven by telegraph noise, Phys. Rev. E 85, 031106 (2012); K. Ouchi, T. Horita, and H. Fujisaka, Critical dynamics of phase transition driven by dichotomous Markov noise, ibid. 74, 031106 (2006).

[26] C. Kim, E. K. Lee, and P. Talkner, Numerical method for solving stochastic differential equations with dichotomous noise, Phys. Rev. E 73, 026101 (2006).

[27] I. Bena, Dichotomous Markov Noise: Exact results for out-ofequilibrium systems (a brief overview), Int. J. Mod. Phys. B 20, 2825 (2006).

[28] I. Bena, C. Van den Broeck, R. Kawai, and K. Lindenberg, Drift by dichotomous Markov noise, Phys. Rev. E 68, 041111 (2003); Nonlinear response with dichotomous noise, 66, 045603(R) (2002); J. M. Porra, J. Masoliver, and K. Lindenberg, Bistability driven by dichotomous noise, Phys. Rev. A 44, 4866 (1991).

[29] J. M. Sancho, Stochastic processes driven by dichotomous Markov noise: Some exact dynamical results, J. Math. Phys. 25, 354 (1984).

[30] A. Morita, Free Brownian motion of a particle driven by a dichotomous random force, Phys. Rev. A 41, 754 (1990).

[31] J. Masoliver, Second-order processes driven by dichotomous noise, Phys. Rev. A 45, 706 (1992); Second-order dichotomous processes: Damped free motion, critical behavior, and anomalous superdiffusion, Phys. Rev. E 48, 121 (1993).

[32] M. O. Cáceres, Computing a non-Maxwellian velocity distribution from first principles, Phys. Rev. E 67, 016102 (2003).

[33] A. Fuliński, Non-Markovian noise, Phys. Rev. E 50, 2668 (1994); Relaxation, noise-induced transitions, and stochastic resonance driven by non-Markovian dichotomic noise, 52, 4523 (1995).

[34] D. R. Cox and V. Isham, Point Process (Chapman and Hall, New York, 1980).

[35] C. Godrèche and J. M. Luck, Statistics of the occupation time of renewal processes, J. Stat. Phys. 104, 489 (2001).

[36] I. McHardy, M. Nizama, A. A. Budini, and M. O. Cá ceres, Intermittent waiting-time noises through embedding processes, J. Stat. Phys. 177, 608 (2019).

[37] For a distribution $W(T)$, the moment generating function can be written as $\hat{W}(u)=\int_{0}^{\infty} d T \exp (-u T) W(T)=$ $\sum_{n=0}^{\infty}\left\langle T^{n}\right\rangle(-u)^{n} / n$ ! where $\left\langle T^{n}\right\rangle \equiv \int_{0}^{\infty} d T W(T) T^{n}$.

[38] With ergodicity of a stochastic processes we mean that its expectation value, correlation, and probability density, in an asymptotic long-time regime, can be obtained from a time average over a single realization of the corresponding indicator functions $[1,2]$. Rigorous mathematical conditions for its validity can be found in Ref. [40].

[39] For each set of chosen parameters, the numerical stationary distribution was obtained by using that $P(v) d v$ equals the fraction of time an arbitrary realization of the process spends in an "infinitesimal" ( $d v)$ neighborhood of $v$. We checked that this procedure (due to ergodicity of the stochastic process) is equivalent to the ensemble probability definition.

[40] R. M. Gray, Probability, Random Processes, and Ergodic Properties (Springer, New York, 2009); S. R. Foguel, The Ergodic Theory of Markov Processes (Van Nostrand Reinhold Company, New York, 1969). 